\title{
EL CAMBIO DE PARADIGMA CIENTÍFICO Y EL DERECHO
}

\section{SCIENTIFIC PARADIGM'S CHANGE AND LAW}

\author{
Martín Rearte*
}

RESUMEN

El objeto de este trabajo es reflexionar sobre el papel que juega el cambio en la concepción de ciencia natural y social, y dentro de esta última, en el Derecho. Comprender la evolución en la ciencia nos puede ayudar a entender por dónde debería proponerse una reforma en la noción de Derecho, a fin de superar la situación de desfase entre distintas instituciones jurídicas con la realidad sobre la que deben operar.

PALABRAS CLAVES: DERECHO * CIENCIAS SOCIALES * CAMBIO * PARADIGMA

\section{ABSTRACT}

The purpose of this article is to reflect on the role played by the changing in the Sciences' —natural and social- meaning; and in the Law Science itself. If we can understand the change in the Science meaning, it could be useful to propose a change in the Law Science meaning. It is the only way to overcome the differences between some law institutions and the reality where they must work.

KEYWORDS: LAW $*$ SOCIAL SCIENCES $*$ CHANGE $*$ PARADIGM

El objeto de este trabajo es reflexionar sobre el papel que juega el cambio en la concepción de ciencia natural y social, y dentro de estas últimas el Derecho, desde una perspectiva epistemológica ${ }^{1}$. Comprender la evolución en la

Doctorando en Derecho Internacional, Universidad Nacional de Cuyo, Mendoza, Argentina. reartemartin@hotmail.com

Entendemos por Epistemología aquella rama de la Filosofía o perspectiva filosófica que reflexiona sobre lo que es conocimiento científico, la ciencia, sus instrumentos y métodos de investigación. Por conocimiento científico, no ciencia nos puede ayudar a entender por dónde debería proponerse una reforma en la noción de Derecho, a fin de superar la situación de desfase entre distintas instituciones jurídicas con la realidad sobre la que deben operar.

necesariamente coincidente con "conocimiento racional" y superador del pensamiento mítico antiguo, lo entendemos caracterizado por ser descriptivo, explicativo y predictivo; crítico y analítico; metódico y sistemático; controlable; unificado; lógico; comunicable; objetivo y provisorio. Puede verse Díaz, Esther (editora). Metodología de las Ciencias Sociales, $1^{\circ}$ edición, Buenos Aires: Biblos, 1997. 
En primer lugar, debemos referirnos a lo que entendemos por paradigmas o, como también han sido llamados, programas de investigación, lenguajes científicos, estructuras lingüísticas o marcos conceptuales ${ }^{2}$. Los paradigmas son considerados, en un primer momento, como realizaciones científicas universalmente reconocidas que, durante cierto tiempo, proporcionan modelos de problemas y soluciones a una comunidad científica. La comunidad científica obtiene con un paradigma un criterio para seleccionar problemas que, mientras se dé por sentado el paradigma, puede suponerse que tienen soluciones. Kuhn sistematizó la noción a través de los elementos o componentes de todo paradigma: los conceptos, las percepciones diferentes entre quienes emplean paradigmas diferentes y por último, los métodos para impulsar la investigación y evaluar sus resultados ${ }^{3}$. Observaremos a continuación el paso gradual del paradigma clásico de ciencia, al reinante a partir del siglo pasado - a través de lo que Khun denomina "revoluciones científicas"-, para analizar luego la recepción obtenida en las ciencias sociales $y$ en el derecho en particular.

\section{LA CONCEPCIÓN TRADICIONAL DE LA CIENCIA MODERNA}

La idea tradicional de la ciencia moderna, se basa en el pensamiento del hombre como

Khun, Thomas S. La estructura de las revoluciones científicas. $1^{\circ}$ ed., $2^{\circ}$ reimp. Trad. Agustín Contín. México: Fondo de Cultura Económica/ Colección Breviarios, 1990. Khun adoptó el término paradigma. Más tarde prefirió adoptar el término de matriz disciplinal para expresar aquello que comparten los miembros de una comunidad científica y que explica la relativa unanimidad de sus juicios profesionales.

Khun, Thomas S. op. cit., p. 197. Son sus componentes: las generalizaciones simbólicas, las creencias en modelos particulares, los valores como la exactitud, las preferencias cuantitativas frente a las cualitativas, el error permisible; $y$ los ejemplares. Esta clase de interpretación podría impedir que se establezcan relaciones deductivas entre teorías rivales. ser racional y la razón como facultad, lo que caracteriza a ese hombre $y$ lo sitúa por encima de los otros seres. El paradigma NewtonianoCartesiano concebido entre los siglos XVI y XVIII, que es el propio de la Física ${ }^{4}$ ("observo para conocer, luego para predecir y en función de ello actúo"), inicia la tensión existente entre ciencias naturales $y$ ciencias sociales. El anhelo del hombre de dominar la realidad $y$ transformarla, propio del Renacimiento, es complementado en el siglo XIX, cuando la humanidad se interesa igualmente por comprender la realidad social.

La ciencia tradicional situó sus cimientos en los conceptos de espacio y tiempo como conceptos absolutos, dimensiones donde acontecen los hechos y por ser inmodificables permiten observar al Universo regido por leyes perfectas. Leyes naturales cuya exactitud permite caracterizar de "estable" a los sistemas, con su correspondiente predecibilidad. El mundo es concebido como un cosmos estable, ordenado por leyes físicas propias de las ciencias causales. Los objetos de las ciencias son ideales, construidos racionalmente, como programaciones perfectas de lo que va a ocurrir. Esto permite a los clásicos introducir las ideas del orden y el equilibrio, que llevan con el tiempo al determinismo científico. Tales conceptos vienen desde la física y la química de la mano de Newton, Descartes y Laplace, pero después son llevados a las ciencias sociales por Tomás Hobbes, quien postula lo ordenado como preferible. El universo es una maquinaria, un mecanismo de relojería - tal como lo entendió Descartes 5 -, formado por partes que interactúan según leyes eternas e inmutables. Este pensamiento pasa también a la política, donde el modelo de Montesquieu de división de poderes

4 De allí que Augusto Comte, en Cours de philosophie positive, haya llamando "Física Social" a la Sociología.

5 Descartes, padre del racionalismo moderno, construye un sistema sobre la base de que cada entidad individual es una máquina que opera por sí sola. Su concepción de un universo mecanístico, en donde tanto lo orgánico como lo inorgánico puede ser definido en términos de extensión y movimiento, y la unidad del mundo físico, significó que se dejaba de lado a la revelación como fuente de la verdad. 
es concebido en términos mecanicistas de un conjunto de partes interrelacionadas.

La comprensión de las sociedades como supraorganismos biológicos, llevó a una idea del Poder derivada de la noción newtoniana de Fuerza. Esta es entendida por los físicos clásicos como la capacidad de operar sobre una cosa para que haga o deje de hacer algo. Este concepto de Poder pasó a las ciencias sociales como la capacidad relacional de condicionar o determinar la conducta de los demás ${ }^{6}$. El relativismo axiológico es propio de esta noción de ciencia, desde Copérnico hasta Descartes ${ }^{7}$. La ciencia entendida como objetiva y axiológicamente neutra, sólo toma los valores como datos o hechos y el observador es neutral o independiente frente a la realidad. Esto se complementa con la idea del egoísmo como motor del progreso y de la competencia como algo natural, la cual fue desarrollada por Adam Smith y luego por el Utilitarismo de Jeremy Bentham y John S. Mill. A partir de entonces el egoísmo, la competencia y la búsqueda de placer constituyeron los pilares axiológicos de la sociedad moderna.

Una de las características centrales del paradigma científico positivista es la idea del reduccionismo. La realidad tiene que ser dividida en tantas partes como sea posible a fin de entender cómo funciona cada una de esas partes. A partir de la comprensión de las partes,

Montbrun, Alberto, "El cambio en la ciencia, el cambio en la política". En: Sociedad vs. Política -Desafios frente al conflicto. Mendoza: Zeta Editores, 2002. Para el autor, este concepto de poder, en las grandes revoluciones liberales, sólo fue contenido, restringido, limitado o reglamentado, fundamentalmente a través de las constituciones escritas, sin alterar su naturaleza intrínseca.

El relativismo axiológico científico es un enfoque de análisis de la realidad que prescinde de la emisión de juicios de valor, fuese eminentemente objetivo y elaborado sobre la base de juicios existenciales o de mero conocimiento. Resulta ser la consecuencia necesaria de la aplicación rigurosa del método científico al análisis de los hechos y fenómenos que conforman la realidad. Se fundamenta en un principio de lógica formal que establece la separación entre el ser y el deber ser. Resulta imposible lógicamente inferir proposiciones de deber ser a partir de proposiciones de ser. es posible integrar el conjunto. La fragmentación del conocimiento no se contradice con los objetivos universales de la ciencia tradicional, para quien la razón es universal. A través de esa razón el hombre conoce y dicta leyes para dominar la realidad. El ego cogito de los clásicos y su logos integrador - la razón entendida como medio para lograr sabiduría, justicia, bondad y verdad- deja su lugar al ego conqueror y su ratio moderna -medio para encontrar la verdad como evidencia, conforme los sentidos-. El fin de la ciencia es, en definitiva, lograr el dominio de la realidad. Observar las regularidades para dictar la ley que las rige.

Aquí se hace evidente la necesidad del método. La metodología dispone las técnicas y procedimientos para la realización de la investigación científica, el camino a la explicación científica del por qué y cómo de los hechos, dada la complejidad de los resultados. La necesidad del método científico se presenta como contexto de justificación, al trabajar el científico con resultados obtenidos en otros trabajos y de allí partir hacia nuevos resultados. Ello permite describir tanto el punto de partida como el por qué de los nuevos resultados obtenidos. El contexto de justificación abarca lo relativo a la validación del conocimiento científico, a la estructura lógica de las teorías y su contrastación empírica.

\section{EL GIRO EPISTEMOLÓGICO}

El cambio, ruptura o giro en este orden de ideas se da principalmente en los últimos cien años, a partir de la llamada crisis del Racionalismo y el fracaso de la ilusión ilustrada. Las críticas versan sobre los postulados epistemológicos de base positivista. Desde el nacimiento del Positivismo como doctrina filosófica, por Augusto Comte ${ }^{8}$, y su derivación

8 Comte considera que la historia de la cultura ha pasado sucesivamente por tres estadíos: teológico, metafísico y positivo. En este último, correspondiente a la modernidad, los fenómenos se explican a partir de las relaciones invariantes que guardan entre sí, las leyes. Se rechaza toda explicación que no pueda verificarse positivamente, por confrontación empírica. 
en las corrientes neopositivistas, principalmente los empiristas y positivistas lógicos del Círculo de Viena ${ }^{9}$, el racionalismo crítico de Popper $^{10}$ y la filosofía analítica de Russell ${ }^{11}$, se postulan coincidencias epistemológicas básicas: la razón humana se reduce a los límites de la racionalidad científica, la necesidad de unificar el lenguaje científico, la determinación de un método común para todas las ciencias y la neutralidad ética del conocimiento científico. Estos serán los principios puestos en tela de juicio.

Las críticas a esta racionalidad positiva no se hacen esperar. Los parámetros establecidos para la ciencia como racionalidad son demasiado estrechos para abarcar la multiplicidad de lo real. En lugar de pensar una racionalidad científica universal e inmutable, la nueva visión de la ciencia apunta a reflexionar teniendo en cuenta su historia, la manera en que los hombres concretos inciden en la aceptación o rechazo de las teorías en cada momento histórico. La ciencia se relaciona con todo el espectro social y cultural como un fenómeno íntegro y no solamente en su aspecto metodológico-formal. La idea de que la reflexión científica debe superar el mero análisis de estructuras vacías para situarse en

El Círculo de Viena se forma en 1929, agrupando a un grupo de filósofos y científicos que tienen como fin fusionar todas las ciencias a partir de la unificación del lenguaje, por medio de incorporar las técnicas y métodos de la lógica matemática al lenguaje. El objetivo es clarificar el lenguaje de la Ciencia.

10 Puede verse Gordillo, Agustín. El método en derecho. Aprender, enseñar, escribir, hacer. Madrid: Civitas, 1988 y $4^{\mathrm{a}}$ reimpresión 2001. Según el autor, el actual momento de la Ciencia - luego de Aristóteles y Hume- se inicia con Popper, el cual no sería acertado llamarlo método hipotético deductivo, porque la hipótesis es siempre singular en el caso del derecho y no existe lugar a deducción alguna a partir de ella.

11 La Filosofía Analítica busca el lenguaje lógicamente perfecto que contara con una simbolización exacta que hiciera transparente la estructura lógica de los hechos. hombres concretos puede situarse en las críticas de Freud ${ }^{12}$, Nietzsche ${ }^{13} y$ Marx $^{14}$.

Los autores plantean con distintos matices sus dudas sobre la calidad de reflejo transparente de la realidad atribuida a la razón. A ello debemos sumar el aporte del llamado giro lingüístico, con el nacimiento de la Semiótica $y$ la Lingüística ${ }^{15}$. La lengua ya no es considerada como una representación fiel, unívoca y denotativa de la realidad, como si se tratase de un vidrio interpuesto ante los hechos. En su lugar signos lingüísticos son ahora abordados como un espejo, interpuesto entre el pensamiento y la realidad, cuyo reflejo puede sufrir oscuridades o deformaciones. Los lenguajes científicos son entendidos como realizaciones científicas universalmente reconocidas que, durante cierto tiempo, proporcionan modelos o criterios para seleccionar problemas y soluciones a una comunidad científica. Con un cambio

Para Freud, las razones más profundas provienen del inconciente, de allí su calidad de irracionales.

Para Nietzsche, la concepción de razón como facultad para conocer y dominar, proviene de la voluntad de saber, como voluntad de poder para lograr la dominación política.

Marx rescata al hombre y sus necesidades económicas, por sobre su caracterización como ser ávido de conocimientos.

A partir del nacimiento de la semiótica como ciencia, la Lengua es considerada como signos, creados por el hombre, dirigidos a un referente, creado socialmente. La palabra es una creación social, cultural, que no necesariamente es una representación de la realidad, resaltando su carácter connotativo. En la manifestación hay una sedimentación en el significado, a través de intereses, necesidades, subjetividades. La semiótica, como ciencia de los signos, estudia los signos dentro del Proceso comunicativo, cuyos niveles son: emisor, receptor, mensaje, referente, dando lugar a los distintos abordajes: Pragmática, Sintaxis y Semántica. El Derecho, como ciencia social, se interesa principalmente por la Pragmática, la relación entre los signos y los usuarios, para descubrir el significado dado por los usuarios a los signos, la recepción por parte de ellos, la intención del emisor. Puede verse Schuster, Félix. El método en las Ciencias Sociales, Buenos Aires: Centro Ed. de América Latina, 1992. 
lingüístico acontece un cambio en el modo en que las palabras determinan sus referentes de la naturaleza. Ese cambio no sólo incide en las nuevas categorías a utilizar, sino en el modo en que los objetos y situaciones dadas son distribuidos entre las categorías preexistentes ${ }^{16}$. A su vez, el llamado giro cultural, introduciendo las nociones de caos, azar, complejidad, contrarias a la idea de Universo como cosmos; también contribuye fuertemente a este cambio en la idea de Ciencia.

\section{LA CONCEPCIÓN ACTUAL DE CIENCIA}

La noción actual de Ciencia, en la que las ramas sociales logran independizarse de las naturales, ya no habla de razón como facultad del hombre, sino de racionalidad ${ }^{17}$. Es decir, la razón en ejercicio, pensando una situación concreta y distinta, y no como vehículo para conocer la Verdad. La ciencia persigue la verosimilitud, como una meta más clara y realista que la búsqueda de la verdad, dado que podemos tener argumentos potentes $y$ razonablemente buenos para pretender haber avanzado hacia la verdad $^{18}$. El cambio de paradigma está marcado

Khun, Thomas S. La estructura de las revoluciones científicas. $1^{\circ}$ ed., $2^{\circ}$ reimp., trad. Agustín Contín, México: Fondo de Cultura EconómicaColección Breviarios, 1990. Si la labor científica consiste fundamentalmente en la construcción de marcos conceptuales, entonces, cómo aceptan los científicos un lenguaje como válido. Para Lakatos por medio del convencionalismo, para Habermas, por medio del diálogo. Para Kuhn, no hay ninguna norma más elevada que la aceptación de la comunidad pertinente.

Habermas, Jurgen, Ciencia y técnica como ideología. Madrid: Tecnos, 1984. El autor distingue la racionalidad estratégica, propia de la actividad científica, de la racionalidad comunicativa, presente en el diálogo de la ética discursiva. En ella desarrolla su Teoría del diálogo y la argumentación, en la búsqueda del argumento más racional y no el que sea funcional a intereses.

18 Popper, Karl, Conocimiento objetivo. Un enfoque evolucionista. Madrid, Tecnos, 1992: 58.

Una teoría tiene más verosimilitud o se aproxima más a la verdad que otra si se siguen de ella más enunciados verdaderos, pero no más enunciados por el cambio en la idea de espacio y tiempo, ya no como conceptos absolutos sino relativos. Esto se complementa por la aceptación de la finitud del universo - a través de los aportes de la Física cuántica-y la inestabilidad de los sistemas, debido a la acción del azar y la casualidad como factores incidentes en los hechos.

Los postulados absolutos de orden y equilibrio son revisados por Einstein y su Teoría de la relatividad, y por la Termodinámica del no equilibrio de Ilya Prigogine ${ }^{19}$. El mundo es desorden, es desequilibrio y es evolución y transformación, dentro de determinados márgenes de estabilidad, salvo cuando se producen bifurcaciones y procesos caóticos. Esto supone también el reconocimiento de la impredictibilidad como una característica de los procesos vitales. Los sistemas vivos operan fuera del equilibrio metabolizando $y$ disipando energía y evolucionando hacia estados más complejos. El universo ficcional del orden ya había sido conmocionado en el siglo XIX con la teoría de los campos magnéticos de Maxwell y Faraday, con la teoría de la evolución de Lamarck y Darwin, con la filosofía de Hegel y la concepción política de Marx, pero recién en el siglo Xx se comprendió cabalmente la naturaleza de los procesos alejados del equilibrio.

El mecanicismo científico es dejado de lado. Los sistemas mecánicos tienen la característica del control externo y no generan autónomamente nuevas capacidades de adaptación al entorno. Deben ser manipulados desde afuera. Los sistemas biológicos, postulado del Organicismo ${ }^{20}$,

falsos o, al menos, igual cantidad de enunciados verdaderos $y$ menos enunciados falsos.

La termodinámica general de Prigogine explica la aparición de "estructuras disipativas" en el mundo físico, las cuales permiten concebir la forma en que el desorden genera orden. Por intermedio de ínfimas fluctuaciones-bifurcaciones se constituyen, a partir del caos y lejos del equilibrio, nuevas organizaciones complejas. Puede verse Spire, Arnaud. El pensamiento de Prigogine. Santiago. Editorial Andrés Bello, 2000.

20 Las concepciones orgánicas que consideran al Estado como un organismo, es decir, una formación natural independiente de la voluntad individual, puede clasificarse en: organicismo filosófico 
se autorregulan y se desarrollan conforme a sus propios componentes y a su capacidad de adaptación al entorno más allá de regulaciones o controles externos. Los problemas deben abordarse desde estrategias integrales $y$ holísticas porque, de no hacerse así, los propios controles internos de los sistemas que se quieren combatir actúan a manera de un subsistema inmunológico y rechazan las intervenciones externas que perciben como amenazantes ${ }^{21}$.

La idea de poder es revisada por la teoría de los sistemas vivos o autopoiéticos ${ }^{22}$. Los sistemas tienen un patrón de red y las redes, por naturaleza, no tienen centro ni control, poniendo en duda la idea de potestad de dirigir procesos. Las modernas teorías de las organizaciones advierten sobre el paso de los liderazgos de mando y autoridad hacia los liderazgos dinamizadores, democráticos y horizontales que operan a partir del ejemplo, del estímulo o de la facilitación. Los sistemas vivos se autoorganizan, son como su propia lógica y su propia deriva los genere, siguiendo la concreta interacción de cada uno de sus subsistemas y de estos con el entorno.

(concibe a la sociedad como una realidad supraindividual, como una unidad de vida originada espontáneamente, sometida a las leyes naturales); organicismo psicosocial (afirma que la sociedad es un organismo supraindividual con ideas, representaciones y mentalidades) y organicismo social (concibe a la sociedad como un organismo biológico, como una realidad vigente $y$ natural, sometida a leyes biológicas que regulan su realidad física, sus procesos vitales de funcionamiento y desarrollo).

21 El organicismo en la actualidad se mantiene a través de las teorías intermedias. Opera políticamente como una teoría de la solidaridad, tanto en el organismo contractual como en el individualismo, en el patriotismo nacionalista, en el colectivismo socialista. El mecanicismo se ha reactualizado con la escuela behaviorista y la teoría instintivista de los hechos sociales y políticos. Montbrun, Alberto, op. cit.

22 Característica de los sistemas vivos de producirse a sí mismos, a través del acoplamiento estructural. Montbrun, Alberto, op. cit.
El relativismo axiológico es revisado por el constructivismo piagetiano ${ }^{23}$. La ciencia no puede prescindir de los valores, no podemos separar al observador de la realidad, el científico condiciona su percepción desde sus propios modelos mentales. Frente al relativismo axiológico y sus posibles consecuencias - el pragmatismo extremo- se acepta la obligación del científico de manifestar los valores de los que parte, a fin de que la transmisión de su conocimiento se reduzca a sus aspectos especulativos. Por otra parte, frente al egoísmo como motor de la historia, los estudios sobre la evolución de las especies vivientes en el planeta contradicen esta idea. Por el contrario, se postula el mecanismo de la simbiosis como el que mejor justifica la permanencia de las especies en el planeta, asociadas intensamente en un proceso eminentemente colaborativo ${ }^{24}$.

Se advierte una fragmentación del reduccionismo científico, señalando que el todo es más -o menos - pero siempre distinto a la suma de las partes. A través de la Gestáltica y la comprensión de la imposibilidad de comprender las partes separadas del todo y postulando una teoría del conocimiento que en vez de orientarse hacia las cosas o los objetos se orienta a las relaciones e interacciones. La Teoría General de Sistemas de von Bertalanffy ${ }^{25}$ y las teorías ecológicas proveyeron de un fuerte sustento científico a la idea de la interrelación y la vinculación entre las diversas ciencias y la necesidad de enfocar la realidad como un todo integrado y no como una discontinua colección de partes aisladas, entendiendo que el sistema actúa y se interrelaciona con su entorno o ambiente. El holismo implica una visión distinta, la realidad no se puede escindir o fragmentar para entenderla, porque la realidad no está hecha de

23 La psicología genética introduce la noción de "asimilación" y la construcción de la realidad en la relación sujeto-entorno. Montbrun, Alberto, óp. cit.

24 Montbrun, Alberto, óp. cit.

25 Bertalanffy, Ludwig von. Teoría General de los Sistemas. $1^{\circ}$ ed. $1^{\circ}$ reimp. Trad. Juan Almeda Madrid: Fondo de Cultura Económica, 1980. 
elementos que interactúan, sino de procesos $y$ de relaciones.

Se supera la concepción clásica o positivista de la ciencia estrechamente ligada a la noción de orden, de la razón, del equilibrio, del control externo. El significado actual de ciencia está relacionado con las ideas de desorden, evolución y caos. La ciencia debe plantearse como objetivo la búsqueda del equilibrio, ya no es posible el razonamiento silogístico en todos los casos y sin miramientos, dado que puede haber otras conclusiones o más de una. Ya no se habla de objetos ideales, sino de regularidades ideales, se construyen regularidades para avanzar en el conocimiento científico. La razón universal y omnisciente abre paso a la crítica de la Razón, tomando las palabras de Kant. Como detrás de toda idea de ciencia se esconde una idea de hombre, el ego conqueror de la ratio moderna - que utiliza la razón como medio para conocer la verdad y dominarla- evoluciona hacia un sujeto sujetado a prácticas sociales ${ }^{26}$ propias de su tiempo y de su momento histórico. El fin de la ciencia es la resolución de problemas, ya sea teórica o en relación directa con la tecnología.

El nuevo pensamiento científico se ocupa de criticar las condiciones de vida existentes, entendiendo esta crítica y consecuente mejora como el fin mismo de la ciencia. El foco es puesto en la Sociedad, ya no en el hombre y se busca la racionalidad en todos los ámbitos de la vida humana, incluido el Arte. El método aquí toma importancia como contexto de descubrimiento, como racionalidad estratégica ${ }^{27}$. No sólo importa si el camino trazado en la investigación es bueno o apropiado, sino saber cómo se llegó a él, desde dónde se hizo ciencia y

Foucault, Michel, La Verdad y las formas jurídicas, México, Gedisa, 1986. También puede verse El orden del discurso, SP, Loyola, 1995 y Las palabras $y$ las cosas. SP, Martins Fontes, 1981. El autor concibe al Derecho como una práctica discursiva, montada en una práctica social. Para conocer la práctica social debo conocer la práctica jurídica, porque es ella la que dice quien tiene la verdad. Sólo excavando en el discurso puedo ver la práctica social y la lucha de Poder detrás, en su concepción del discurso como técnica de Poder. el entorno político del científico. El contexto de descubrimiento comprende la manera en que el científico arribó a sus afirmaciones, en función del medio en el que llevó a cabo su trabajo, su devenir personal, sus relaciones de poder $y$ sus aspiraciones. Ningún método es considerado neutro.

\section{CONSECUENCIAS DEL GIRO EPISTEMOLÓGICO}

Las consecuencias de este giro epistemológico pueden percibirse en la caída de algunos mitos. La autonomía del pensamiento científico, la idea de la comunidad científica como ajena a la estructura social, sus intereses y necesidades. El conocimiento no parte nunca de cero, sino que siempre presupone un conocimiento que se da por supuesto en un momento determinado junto con un problema planteado. Habermas nos habla de la conexión entre conocimiento e interés, los cuales operan como orientaciones o estrategias cognoscitivas generales de los distintos tipos de investigación ${ }^{28}$. De igual forma cae la idea de que todo lo ajeno a la ciencia es irracional. El cambio postula considerar todas las formas de ejercer la racionalidad, $y$ al hecho científico en relación con la base productiva, el sistema de necesidades y

28 Habermas, Jurgen, óp. cit., p. 169 a 171. Los procesos de investigación se clasifican en tres categorías: ciencias empírico-analíticas, que comprenden las ciencias de la naturaleza y las ciencias sociales; las ciencias histórico-hermenéuticas, que comprenden las humanidades y las ciencias históricas y sociales; $y$ las ciencias de orientación crítica, que abarcan la crítica de la ideología (teoría social crítica). Para cada una de estas categorías de investigación, se postula una conexión con un interés cognoscitivo específico: un interés cognoscitivo técnico; práctico; y emancipatorio, respectivamente. La primera persigue un interés por la seguridad informativa y ampliación de la acción de éxito controlado, mediante el método empírico-analítico para lograr un saber-predicción. La segunda obtiene sus conocimientos de la comprensión de sentido. Las últimas se basan en la reflexión crítica para lograr la organización de las relaciones humanas sobre la base de una intersubjetividad no coaccionada, dando lugar a sus Éticas dialógica y formal. 
las estructuras de Poder. Se hace ciencia por curiosidad, pero también $-y$ sobre todo- por intereses, ideología y Poder.

El cambio de enfoque es consecuencia de esta nueva interpretación. Desde los "objetos" o las "cosas" a las relaciones y procesos de transformación que se verifican entre ellos. De lo que los sistemas "son", es decir, de las descripciones ontológicas, a lo que los sistemas "hacen", lo que se traduce en descripciones epistemológicas. Con respecto a la verdad, un cambio de actitud, reemplazando la búsqueda de su conocimiento por la comprensión aproximada del proceso. Para Popper, cuando el científico contrasta dos teorías, puede resultar que una Teoría sea aceptable, o verificada, o bien falsada. Pero en ningún caso puede hablarse de verdadera $^{29}$. La tarea de la investigación consiste en las contrastaciones sistemáticas a que debe someterse toda idea nueva antes de que se la pueda sostener sistemáticamente, pero subrayando que no existe lógica de la verificación, pero sí existe lógica de la refutación ${ }^{30}$.

El sistema científico presenta ahora una ruptura con la fragmentación disciplinaria clásica de las ciencias. Pero quizás el mayor cambio está dado por la concepción del Sujeto: el observador es parte de la realidad observada $y$

Popper, Kart. La lógica de la investigación científica. $1^{\circ}$ ed. $11^{\circ}$ reimp. Trad. Victor Sánchez de Zavala. Madrid. Tecnos, 1973: 33. "En ningún momento he asumido que podamos pasar por un razonamiento de la verdad de enunciados singulares a la verdad de teorías. No he supuesto un sólo instante que, en virtud de unas conclusiones verificadas, pueda establecerse que unas teorías sean verdaderas, ni siquiera meramente probables".

30 Desde un punto de vista estrictamente lógico, nunca podemos afirmar que una hipótesis es necesariamente cierta porque esté de acuerdo con los hechos; al pasar en nuestro razonamiento de la verdad de los hechos a la verdad de la hipótesis, cometemos implícitamente la falacia lógica de afirmar el consecuente. Por otra parte, podemos negar la verdad de una hipótesis en relación con los hechos, porque, al pasar en nuestro razonamiento de la falsedad de los hechos a la falsedad de la hipótesis, invocamos el proceso de razonamiento, lógicamente correcto, denominado negar el consecuente. no ajeno a ella. Se presenta una unidad entre sujeto-objeto, superando la separación del sujeto delimitador del objeto, que está fuera de él. El sujeto vive el fenómeno, lo experimenta a la vez que lo explica. Los antipositivistas dirán que lo comprende, no que lo explica; siendo esta comprensión desde adentro del fenómeno social. Es necesario destacar la importancia dada al discurso en la nueva ciencia. Ella es entendida principalmente como práctica social ${ }^{31}$ - que no puede entenderse separada de su carácter discursivo ${ }^{32}$, connotativo, persuasivo $y$ en constante lucha con lo dictado por teorías anteriores en busca de imponer "su" verdad. Aquí nuevamente la obra de Foucault es reveladora ${ }^{33}$, al igual que la de Thomas Khun ${ }^{34}$.

Díaz, Esther (comp.). La producción de los conocimientos científicos. Buenos Aires: Biblos, 1996.

Schuster, Felix, op cit.

Foucault, Michel, op. cit. La ciencia es una práctica occidental que busca el establecimiento de la verdad. Dentro de ellas, la práctica jurídica es de gran importancia ya que busca la verdad de lo ocurrido, la verdad jurídica, pasando de la infracción moral a la jurídica. Las prácticas jurídicas, propias de las sociedades complejas y estructuradas, se transfieren a las prácticas científicas y discursivas, que legitiman lo que el sistema jurídico ha legalizado. Para el autor, sabremos como valida la verdad una sociedad, al ver como esa misma sociedad sanciona las infracciones. Los operadores del derecho imponen su forma de validar la verdad a la ciencia. Las prácticas Jurídicas, al estatizarse el Derecho, disminuyen el número de individuos capaces de decirlo, a través de la selección de personas especializadas para ello, lo que se corresponde a una división de roles sociales. Lo mismo ocurre con las prácticas científicas, donde es limitado el número de personas que pueden validar una verdad. A su vez, el Derecho es una práctica social privilegiada, por su cercanía con el poder, lo que lo convierte en un modelo para otras ciencias sociales y en un medio de control de conductas. Como consecuencia de ello, la sociedad asume ese modelo como natural, como lo que debe ser, como el estado máximo de progreso. El estado, resultado de un proceso de lucha de poderes, es la manifestación del discurso del vencedor de esa lucha.

34 Khun, Thomas S. La estructura de las revoluciones científicas, $1^{\circ}$ ed., $2^{\circ}$ reimp. Trad. Agustín Contín, México: Fondo de Cultura Económica-Colección 
Para hablar del nuevo paradigma para el pensamiento occidental, debemos referirnos a la obra de Ilya Prigogine ${ }^{35}$. El autor invita a superar la contradictoria idea de que el universo está regido por leyes deterministas $y$, sin embargo, los hombres se mueven y actúan libremente en él, por lo que propone desarticular el determinismo (o raciocinio cerrado) de las ciencias mediante una perspectiva de las probabilidades $y$ de las posibilidades evolutivas. La naturaleza - para Prigogine - es creativa, inventa constantemente y su dimensión temporal está muy lejos de agotarse en la idea matemática cartesiana de un tiempo absoluto. Prigogine articuló lo que podríamos llamar una nueva certidumbre: el futuro no está escrito en ninguna parte. Concede al tiempo - por lo tanto-su creatividad fundamental $y$ a los hombres su responsabilidad intrínseca en la construcción del porvenir. Lo que pretende es encontrar el nexo entre un universo determinista -que es alienante-y el universo aleatorio - donde no habría lugar para la razón con los límites que le asigna el racionalismo clásico-.

La vocación humanista de Prigogine es quizás la que mejor define esta nueva ciencia. Sus ideas en el marco de las Ciencias Formales o exactas, había sido anticipada por Popper, y su crítica al determinismo en términos metodológicos: "la ciencia supone no adorar al ídolo de la certidumbre (...) La opinión equivocada de la ciencia se detalla en su pretensión de tener razón: pues lo que hace al hombre de ciencia no es su posesión del conocimiento,

Breviarios, 1990. Al referirse a las causas de las revoluciones científicas, distingue en primer lugar el descubrimiento de la anomalía - la naturaleza ha violado las expectativas, inducidas por el paradigma que rigen la ciencia normal- Se trata de un proceso de asimilación conceptual, de creación o reformulación de un lenguaje. Además del cambio de paradigma por descubrimiento, también puede operar como resultado de la formulación de nuevas teorías, cuando fallan los esfuerzos de articulación de los paradigmas existentes a las anomalías reconocidas.

Prigogine, Ilya. El fin de las certidumbres. Barcelona, Crítica: 2000. También puede verse Spire, Arnaud, óp.cit. de la verdad irrefutable, sino su indagación de la verdad [realidad] persistente $y$ temerariamente crítica". Para Popper, la ciencia, la nueva ciencia, no debe perseguir "la ilusoria meta de que sus respuestas sean definitivas, ni siquiera probables; antes bien (...) la de descubrir incesantemente problemas nuevos, más profundos y más generales $y$ de sujetar nuestras respuestas (siempre provisionales) a contrastaciones constantemente renovadas y cada vez más rigurosas" ${ }^{36}$. Ambos rescatan, en definitiva, al hombre como hacedor libre de su propia realidad.

\section{EL CAMBIO DE PARADIGMA EN LAS CIENCIAS SOCIALES, Y EN EL DERECHO}

La cientificidad de las Ciencias Sociales siempre fue cuestionada, conforme los parámetros de la que hemos denominado concepción tradicional de ciencia, en cuanto a su objetividad, neutralidad valorativa y su capacidad para el establecimiento de leyes, entendidas en el sentido clásico de leyes que describen, explican y predicen. La diferenciación apunta al método científico: las naturales utilizan el hipotéticodeductivo, dando lugar a una explicación causal, mientras las sociales el dialéctico, siendo la explicación a la que arriban intencional o funcional $^{37}$. Popper introduce la noción de aducción para superar la dificultad inductiva en las

36 Popper, Karl, La lógica de la investigación científica. Trad. Victor Sánchez de Zavala. $1^{\circ}$ ed., $11^{\circ}$ reimp. Madrid. Tecnos, 1973: 260-61.

Popper, Karl, óp. cit., Popper va a combatir esta pretendida dicotomía entre Ciencias Naturales y Sociales, afirmando que el método en ambas es el mismo. El método siempre consiste en ofrecer una explicación causal deductiva por medio de predicciones. Pero sí observa una diferencia en la dificultad de aplicar determinados métodos en las Ciencias Sociales. Tales como el método de la construcción racional o lógica, de construir un modelo con base en una suposición de completa racionalidad e información por parte de todos los individuos implicados, $y$ luego de estimar la desviación de la conducta real de la gente con respecto a la conducta modelo, usando esta última como una especie de coordenada cero. 
ciencias sociales. Ante argumentos inductivos que tratan de demostrar que una determinada hipótesis se ve apoyada por determinados hechos - siendo argumentos "no demostrativos", es decir, que no se deducen de las premisas mayor y menor- el autor nos habla de operación no-lógica de la aducción. Ella nos permite saltar del caos que es el mundo real a la corazonada que supone una conjetura. La cuestión de cómo se produce dicho salto pertenece al contexto de la lógica del descubrimiento.

Más allá del aspecto metodológico, la incorporación de la idea de comprensión propia del sujeto que vive el fenómeno que estudia -en reemplazo de la fase de explicación del sujeto separado de su objeto o hecho neutro valorativamente - nos permite adentrarnos en la perspectiva de la Fenomenología ${ }^{38}$. Las ciencias sociales estudian fenómenos, esto es, lo que se le aparece al sujeto, que explica y comprende, sin dejar de incorporar en ellos sus propias percepciones ${ }^{39}$. Aquellos son significativos, tienen sentido para el sujeto y los manifestados en el orden social tienen un predicado axiológico dado por la propia perspectiva del sujeto, superando la noción de hechos valorativamente neutros. Los fenómenos tienen sentido, deben ser interpretados por el investigador.

La crítica fenomenológica no tardó en apuntar al Derecho como ciencia, ya sea como ciencia social -Ciencia Jurídica- o como práctica social -Derecho-. Ocupada

Husserl, Edmund, Ideas relativas a una fenomenología pura y una filosofía fenomenológica. México: Fondo de Cultura Económica, 1962. La interdependencia del sujeto con el mundo se da en una constante tensión entre el sujeto y su objeto, sin poder pensar uno sin el otro, por lo que todo fenómeno se da en el mundo o en lo social.

Ver también Schuster, Felix. Ciencia y presuposiciones. Buenos Aires. Eudeba, 2000. El autor distingue, entre los denominados presupuestos del investigador, los ejes ontológicos (mundo), metodológicos (método), lógico (reglas de inferencia), conceptual (términos) y axiológicos (valores). Todos ellos dentro de las dimensiones social específica (contextos de descubrimiento y validación) y social general (contextos de producción y aplicación). principalmente de la pragmática del discurso ${ }^{40}$, $y$ dentro de las ciencias donde la conducta $y$ ética del científico es de alta pertinencia ${ }^{41}$. Su objeto de estudio se centra en hechos y no en signos o símbolos como las Ciencias Formales. Sólo se sirve de ellos como instrumentos para plasmar sus enunciados, que son sintéticos con definiciones en parte descriptivas $y$ en parte normativas. El método de verificación o validación es inductivo, previa constatación con los hechos de la experiencia y no responde necesariamente a un sistema lógico axiomático, donde las reglas se deducen en todos los casos de principios rectores. Aquí radica la crítica principal a la noción del Derecho en nuestro tiempo: una ciencia Social, con un pretendido método silogístico de ciencia Formal.

Dentro de la Teoría Crítica y aquellas que presentan al Derecho Estatizado como una forma de contrastar $y$ validar conocimientos ${ }^{42}$, también se pone en duda la pretendida simetría existente entre Derecho y Ciencia, al criticar la Explicación Científica Jurídica como método normológico deductivo y la sentencia como un mero silogismo. El método formal aplicado al Derecho pretende trazar una simetría entre la explicación científica y la sentencia judicial. En el primero, la deducción lógica extraída del silogismo deductivo permite un razonamiento demostrativo a partir de enunciados de condiciones generales, leyes generales, y la consecuente explicación o descripción del fenómeno que se explica. La conclusión, dada por

Por oposición a las ciencia formales, de baja pertinencia, donde la honestidad del científico no afecta el resultado obtenido.

42 Samaja, Juan. Epistemología y Metodología. Buenos Aires: Eudeba, 1994. Para el autor, a través de la sistematización del Derecho se transfiere la forma de contrastar contenidos deductivamente, y no a la inversa. El estado, sistematizado, sostiene contenidos como derivados de una matriz jurídico-estatal. La verdad, obtenida deductivamente a través de normas estatales, se obtiene de casos particulares, lo que supone una forma de validar esa verdad. Esta forma de contrastación y validación científica, el procedimiento científico, tiene su origen en la estructura jurídica-política del estado, y desde allí se traslada a la ciencia. 
la descripción del fenómeno, permite predecir futuros fenómenos de similares características. En el caso de la sentencia judicial, las circunstancias del caso y las leyes dadas a priori por la estructura legal, funcionan como "explanans", siendo la deducción lógica del veredictum el explanandum correspondiente ${ }^{43}$.

¿Por qué el método formal silogístico no es aplicable a la ciencia jurídica? Porque la necesidad de dictar leyes causales y universales acerca de las relaciones entre fenómenos, propios de la física newtoniana, no es extensible a las Ciencias Sociales. Ellas no tratan de determinismos causales exactos, sino de situaciones conformadas por relaciones complejas de fuerzas interactuantes. Estudian al hombre no solamente como ser biológico, sino también como poseedor de libertad, capaz de tomar decisiones; inconsciente que lo sujeta a impulsos y hacedor de su propia cultura a través del lenguaje. El Derecho, en tanto práctica social histórica, se valió del método formal silogístico en un determinado momento histórico, lo que no quiere decir que el método responda satisfactoriamente a una realidad totalmente distinta como la de hoy. Es más, es consecuencia de ese mismo tipo de pensamiento lógico lo que no lleva a creer

Cossio, Carlos. La función social de las escuelas de abogacía. Buenos Aires. Instituto Argentino de Filosofía Jurídica y Social. 1945, ps. 100 y ss. La Teoría Egológica del Derecho se gestó en el seno de la "tercera generación" de pensadores latinoamericanos del siglo XX, quienes se esforzaron por pensar y repensar los problemas desde América Latina, $y$ tratando de encontrar soluciones no con pautas preestablecidas sino con recursos originarios. Para la Egología, el Derecho es la conducta de los hombres, es aquella misma libertad con la que unos interfieren o pueden interferir en la conducta de los otros. Esta tarea ha sido mostrada como la que media entre el ser propio de la naturaleza y el deber ser característico del Derecho y de la Moral. Hasta la aparición de Cossio en la escena histórica, esta diferencia aparece como categoría mental (tal como lo podemos encontrar en Kant y en Kelsen). Desde las categorías inauguradas por la filosofía existencial, la Egología señala una diferencia en el objeto y no de orden meramente conceptual entre las ciencias jurídicas y las ciencias naturales, siendo la vida humana en su libertad, para las primeras y la vida biológica, para las segundas. que un cambio en el contenido normativo de un determinado conflicto cambiará la práctica social involucrada.

Dijimos que el derecho es entendido tanto como ciencia social - Ciencia Jurídicay como práctica social —Derecho- Cuando nos referimos al método en la Ciencia Jurídica, nos referimos al ejercicio del Derecho para producir conocimiento científico, entre otros el funcionamiento y legitimación del sistema normativo y las decisiones judiciales, a través de las sentencias. Ellos son objetos de estudio científico, y no sólo cuestiones propias de la coyuntura política. En cambio, al expresarnos por el Derecho entendemos por él a la práctica social que incluye a todos los operadores jurídicos con una fuerte carga semántica ${ }^{44}$ en la expresión: Derecho como sistema de normas reguladoras de lo Social. El problema se plantea cuando el operador jurídico hace Derecho y busca validar su resultado como si hiciera Ciencia Jurídica y además aplicando el método formal silogístico propio de las ciencias naturales.

La Ciencia Jurídica, ante la carencia de investigación empírica sobre el real funcionamiento de las instituciones jurídicas que la caracterizó durante mucho tiempo, se ocupó de la traslación de modelos jurídicos de interpretación como métodos de investigación, especialmente el exegético-deductivo. Esto llevó a la caracterización del ordenamiento jurídico como sistema, resaltando la estructura simétrica de la sentencia con la explicación científica silogística. La Ciencia Jurídica tenía así como único fin superar problemas de indeterminación o ambigüedad, así como la superación de las lagunas jurídicas. Esto llevó principalmente al desarrollo de teorías de la argumentación y a aquellas centradas en el Derecho como discurso.

Dentro de la perspectiva crítica actual se habla, como hemos dicho, de Derecho como práctica social y como discurso además del sistema normativo positivo. El fin de esta visión es

Schuster, Felix, óp. cit. Semántica entendida como el abordaje de la Semiótica referido a la relación entre los signos y el mensaje con el referente, lo que permite ver la verdad o falsedad del mensaje, en función de la fidelidad de su representación de la realidad. 
armar categorías teórico-epistemológicas que den cuenta del anclaje del Derecho en formas históricas de sociabilidad y en las instituciones. Dichas categorías, lejos de trabajar exclusivamente en forma intrasistémica como en la concepción anterior, da cuenta de los lazos extrasistémicos entre el Derecho y factores políticos, sociales y económicos. Las ciencias sociales $-y$ entre ellas el Derecho- deben considerar a lo inmediatamente dado como algo sospechoso $y$ no como prueba de lo que realmente pasó ${ }^{45}$.

\section{REFLEXIONES FINALES}

Para poder decir que el Derecho como ciencia social ha acompañado el giro epistemológico evidenciado en las demás ciencias sociales, debe superar el paradigma clásico de orden absoluto-relativismo axiológico y, sobre todo, el reduccionismo normológico tan común entre los operadores del Derecho. Aunque también deberá evitar cualquier otro reduccionismo ya sea discursivo o sociologizante. El fenómeno jurídico puede ser abarcado como Sistema de Normas, universal y racional, aunque dicha perspectiva deja de lado la que el Derecho es, ante todo, un producto histórico-social, resultado de un proceso histórico no lineal, de una lucha de poderes ${ }^{46}$. Puede ser abarcado como discurso, como una mediación simbólica a través del lenguaje, que como tal no es aséptico, sino cargado de intereses e ideología. O bien puede ser concebido el Derecho como una práctica social producto de la sociedad y su historia siempre cercano a las relaciones de poder y que involucra a todos los operadores del Derecho, no sólo a los legisladores y jueces. La apreciación que tengamos del fenómeno jurídico incidirá directamente en lo que entenderemos por objeto del Derecho; es decir, por aquella materia de la cual el Derecho se ocupa.

La reformulación del objeto del Derecho ha sido encarada de la Ciencia Jurídica por las

Ortiz, Renato. Taquigrafiando lo social. Argentina: Siglo XXI, 2004.

Foucault, Michel, óp. cit. llamadas corrientes integradoras. El punto de partida en este cambio de enfoque jurídico puede ser hallado en la Teoría Egológica del Derecho formulada por Carlos Cossio ${ }^{47}$. Frente a la concepción clásica del Derecho visto como sistema de normas, la escuela egológica lo ve como comportamiento humano social e interindividual, cuyo sentido axiológico se encuentra expresado conceptualmente por las normas jurídicas vigentes en la comunidad. Dentro de esta nueva visión se enmarcan otras corrientes latinoamericanas, como el Tridimensionalismo de Miguel Reale, al Indivitrialismo de Miguel Herrera Figueroa y al Trialismo de Werner Goldschmidt. Refiriéndose a estas corrientes de pensamiento, fue precisamente Goldschmidt, quien distinguió los pluridimensionalismos de los unidimensionalismos dikelógicos, normológicos y sociológicos ${ }^{48}$. Sobre cada uno de ellos, podemos decir brevemente que el reduccionismo dikelógico afirma que el Derecho está compuesto sólo por exigencias de justicia objetivas - tanto el iusnaturalismo católico de Tomás como el protestante de Grocio y Pufendorf-, el reduccionismo positivista identifica el Derecho con un conjunto de normas humanas, arrancando el Derecho Natural y las consideraciones de justicia de la ciencia jurídica - tanto imperativistas como los partidarios de la Escuela del Derecho Puro de Kelsen-y por último el reduccionismo sociológico encuentra al Derecho en la realidad social en su dimensión psicofísica, más precisamente en el accionar de los operadores del derecho - Jerome Frank en su versión norteamericana, y Olivecrona entre los escandinavos-.

Como nos enseña Goldschmidt, la Ciencia Jurídica debe superar las limitaciones de cada uno de los reduccionismos, planteándose la tarea de salir del extremismo iusnaturalista, del formalismo kelseniano y del conservadurismo

Cossio, Carlos. La teoría egológica del derecho y el concepto jurídico de libertad. Buenos Aires: Losada, 1964.

Goldschmidt, Werner. "Tridimensionalismo, realismo genético y justicia. Homenaje a Miguel Herrera Figueroa”. En Revista La Ley 1983-A, 755. 
sociologizante. ¿Por qué la necesidad de superar la visión unidimensional del Derecho? El cambio paradigmático de la Ciencia, y en especial de las Ciencias Sociales, ha demostrado que el conocimiento científico es hijo de su tiempo. El pensamiento positivista, aún enseñado en nuestras universidades como única forma de razonamiento jurídico, tuvo, en palabras de Goldschmidt, "una vida relativamente tranquila mientras el mundo parecía consolidado a base de movimientos triunfantes en lo espiritual, el cristianismo; en lo económico-social, el capitalismo burgués; en lo político, el régimen demo-liberal" ${ }^{\prime 9}$. Su crítica a través de las teorías que hemos llamado integradoras, aparece de la mano de una serie de fenómenos socio-políticos, como el comunismo soviético, los fascismos europeos, las democracias populares y el ateísmo; que ponen en tela de juicio esta pretendida escisión de la realidad social y los valores de la ciencia jurídica. Una reacción se refugia en lo que denominamos el reduccionismo dikelógico, retornando al derecho natural. Otra, busca recuperar los aspectos olvidados del Derecho, e integrarlos.

El Derecho como conocimiento científico debe dar el salto cualitativo desde el paradigma formal clásico —o "reduccionista" — hacia uno integral que permita abarcar en su objeto de estudio tanto hechos, normas y valores. Es decir, diferenciar las tres dimensiones $y$ al mismo tiempo integrarlas en una complejidad pura, superando la purificación metodológica de Kelsen, y la simplificación de los reduccionismos unidimensionales. Ello nos permitiría analizar rigurosamente aspectos olvidados por la ciencia jurídica, que en su afán de purificarse, se ha descuidado de la complejidad del mundo jurídico acorde a la importancia de su objeto: la vida humana misma ${ }^{50}$.

49 Goldschmidt, Werner. Introducción al derecho, $2^{\circ}$ Edición. Buenos Aires. Aguilar, 1962: 41.

50 En Goldschmidt, Werner, óp. cit., se propone abarcar el objeto de la Ciencia Jurídica como una complejidad pura, por oposición a la simplicidad pura del Derecho pretendida por Kelsen, y la complejidad impura de la teorías que distinguen pero mezclan sus elementos, en lugar de integrarlos.
La vocación humanista de la que hablamos tiene su corolario en el objeto de las Ciencias Sociales y del Derecho. Es la hombre, el sujeto en su dimensión social como una integridad biológica, psicológica, espiritual y social. El hombre en su dimensión cultural, como resultado de la cultura que él mismo crea, de las decisiones que él mismo toma y, a la vez, sujeto sujetado a prácticas sociales, productor de lenguaje y de sistemas simbólicos, y sujeto a impulsos involuntarios. El objeto del Derecho no puede limitarse a un mecanismo formal silogístico, no puede rastrearse sólo en las normas o en la actividad de los jueces, o centrarse en catalogar de justas o no esas normas y esas actividades. El objeto del Derecho es todo eso junto, por lo que, en conclusión, el objeto del Derecho no puede ser reducido a normas. Aceptar ello y su consecuente metodología formal silogística significa escindir al Derecho del cambio paradigmático que ha desarrollado la Ciencia en General y las Ciencias Sociales en particular en el último siglo.

\section{BIBLIOGRAFÍA}

Bertalanffy Ludwig von. Teoría General de los Sistemas. $1^{\circ}$ ed., $1^{\circ}$ reimp. Trad. Juan Almeda. Madrid: Fondo de Cultura Económica, 1980.

Cossio, Carlos. La función social de las escuelas de abogacía. Buenos Aires: Instituto Argentino de Filosofía Jurídica y Social, 1945 , ps. 100 y ss.

La teoría egológica del derecho y el concepto jurídico de libertad. Buenos Aires: Losada, 1964.

Díaz, Esther (editora). Metodología de las Ciencias Sociales. $1^{0}$ edición. Buenos Aires: Biblos, 1997.

Goldschmidt, Werner. "Tridimensionalismo, realismo genético y justicia. Homenaje a 
Miguel Herrera Figueroa”. Revista La Ley 1983-A, 755.

. Introducción al derecho. $2^{\circ}$ Edición. Buenos Aires. Aguilar, 1962: 41.

Foucault, Michel. La verdad y las formas jurídica. México: Gedisa, 1986.

Gordillo, Agustín. (1988). El método en derecho. Aprender, enseñar, escribir, hacer. $4^{a}$ reimpresión. Madrid: Civitas, 2001.

Habermas, Jurgen. Ciencia y técnica como ideología. Madrid: Tecnos, 1984.

Husserl, Edmund. Ideas relativas a una fenomenología pura y una filosofía fenomenológica. México: Fondo de Cultura Económica, 1962.

Khun, Thomas S. La estructura de las revoluciones científicas. $1^{\circ}$ ed., $2^{\circ}$ reimp. Trad. Agustín Contín. México: Fondo de Cultura Económica-Colección Breviarios, 1990.
Montbrun, Alberto. "El cambio en la ciencia, el cambio en la política". Revista Sociedad vs. Política-Desafíos frente al conflicto. Mendoza: Zeta Editores. 2002.

Ortiz, Renato. Taquigrafiando lo social. Buenos Aires: Siglo XXI, 2004.

Popper, Kart. La lógica de la investigación científica. $1^{\circ}$ ed., $11^{\circ}$ reimp. Trad. Víctor Sánchez de Zavala. Madrid: Tecnos, 1973.

Prigogine, Ilya. El fin de las certidumbres. Barcelona: Crítica, 2000.

Samaja, Juan. Epistemología y metodología. Buenos Aires: Eudeba, 1994.

Schuster, Felix. El método en las Ciencias Sociales. Buenos Aires: Centro ed. de América Latina, 1992.

Ciencia y presuposiciones. Buenos Aires: ed. Eudeba, 2000.

Spire, Arnaud. El pensamiento de Prigogine. Santiago de Chile: editorial Andrés Bello, 2000. 